\title{
Traduire
}

Revue française de la traduction

$220 \mid 2009$

Organisations internationales | Bicentenaire de Louis Braille

\section{La traduction juridique dans le contexte international ou l'art du compromis}

L'exemple du Statut de la Cour pénale internationale

\section{Véronique Sauron}

\section{OpenEdition \\ Journals}

Édition électronique

URL : http://journals.openedition.org/traduire/376

DOI : 10.4000/traduire.376

ISSN : 2272-9992

\section{Éditeur}

Société française des traducteurs

Édition imprimée

Date de publication : 15 juin 2009

Pagination : 14-23

ISSN : 0395-773X

Référence électronique

Véronique Sauron, « La traduction juridique dans le contexte international ou l'art du compromis »,

Traduire [En ligne], 220 | 2009, mis en ligne le 12 novembre 2013, consulté le 01 mai 2019. URL : http:// journals.openedition.org/traduire/376; DOI : 10.4000/traduire.376 


\title{
La traduction juridique dans le contexte international ou l'art du compromis
}

\author{
L'exemple du Statut de la Cour pénale internationale
}

Véronique Sauron

\section{Introduction}

II n'est pas rare d'entendre les traducteurs (spécialisés ou non dans le domaine juridique) se moquer ou se plaindre - c'est selon - de la qualité des traductions produites au sein des institutions et organisations régionales ou internationales. Combien de fois n'a-t-on assisté à des discussions fustigeant le manque de rigueur et de précision dans la terminologie juridique retenue ou le caractère flou et jargonisant de telle ou telle formulation rencontrée dans un texte international et prônant la retraduction des textes existants. Si les critiques formulées peuvent, dans certains cas, apparaître légitimes, elles n'en sont pas moins le plus souvent le reflet d'une méconnaissance de la manière dont la traduction est envisagée et effectuée au sein de ces institutions ${ }^{(1)}$ et de la nature même du droit international qui s'analyse en un ensemble de normes régissant le comportement des États, que ces derniers négocient et concluent entre eux. Ce caractère négocié est probablement ce qui distingue le plus les instruments internationaux des autres textes juridiques et explique, dans une large mesure, les choix de traduction qui sont effectués. Les âpres discussions auxquelles la création de la Cour pénale internationale (CPI), institution originale qui marque un compromis entre les systèmes de Common Law et de droit romano-germanique, fournit un exemple intéressant des difficultés qui peuvent résulter de la confrontation au niveau international de deux systèmes juridiques réputés très différents, des choix terminologiques qui sont faits et partant, des contraintes qui pèsent sur ceux qui sont appelés à traduire les solutions négociées par les États. Après un rappel des circonstances qui ont présidé à la création de la Cour et une présentation de ses principaux organes, nous analyserons, à la lumière des divers documents publiés dans le cadre des travaux préparatoires à la Conférence des plénipotentiaires, quelques concepts de droit (et leur traduction) présents dans le Statut de la CPI qui ont fait l'objet de compromis, prenant par là même une signification différente de celle qu'ils peuvent avoir dans les systèmes juridiques nationaux.

(1) Voir "Stratégies normalisatrices et culture institutionnelle : un regard sur la traduction dans les organisations internationales ", communication présentée par Véronique Sauron et Maite Aragonés Lumeras à l'occasion du $1^{\text {er }}$ colloque de traduction spécialisée, La Havane, Cuba, 8-13 décembre 2008. 


\section{La création de la Cour pénale internationale : un siècle d'hésitations}

La guerre a été et demeure le théâtre des exactions les plus graves et de la commission des crimes les plus odieux, une paix durable ne peut être conclue et consolidée que si les auteurs de ces crimes, du dirigeant gouvernemental au simple exécutant, doivent rendre des comptes devant la justice. La création de la Cour pénale internationale, le 18 juillet 1998, a donc marqué une étape importante dans l'histoire du droit international, celle de la fin de l'impunité pour les auteurs et les instigateurs des crimes les plus graves contre le droit international. II aura fallu plus de cinquante ans pour concrétiser ce vœu d'une cour pénale internationale permanente qui soit à même de répondre aux exigences fondamentales d'humanité.

La communauté internationale avait, à la fin du siècle dernier, pris conscience de la nécessité de mettre en place une instance judiciaire appelée à défendre et à mettre en œuvre les exigences profondes de l'humanité. La communauté internationale avait, en 1899 à La Haye, proclamé pour la première fois la nécessité de répondre à ces exigences. Un pas fut franchi, après l'ampleur des crimes perpétrés par le régime nazi pendant la Seconde guerre mondiale et les exactions commises par les forces japonaises, avec la mise en place des tribunaux de Nuremberg et de Tokyo, chargés de juger les responsables de ces crimes de guerre et crimes contre l'humanité. Ce n'est toutefois qu'après 1945 et en partie sur la base des travaux de ces deux tribunaux, notamment en ce qui concerne la définition des crimes contre l'humanité, qu'une base juridique allait être progressivement créée et intégrée dans des conventions internationales mais aussi dans nombre de législations internes.

En dépit de la construction progressive d'un socle juridique, on ne pouvait prétendre à l'instauration d'une institution judiciaire internationale permanente. Certes, dans le cadre des tribunaux de Nuremberg et de Tokyo, plusieurs États avaient démontré de manière concrète leur implication dans la répression des crimes qui portaient atteinte à l'humanité toute entière. Pour autant, il s'agissait plus de la justice des vainqueurs contre les vaincus que d'une véritable justice internationale. La tendance n'a fait que se confirmer par la suite : la répression des crimes les plus graves était souvent circonscrite aux crimes commis dans des conflits armés internationaux, les tribunaux nationaux étant appelés à juger les criminels de moindre envergure. L'instauration de deux tribunaux spéciaux ad hoc après les massacres perpétrés dans l'ex-Yougoslavie et au Rwanda s'inscrit également dans cette perspective. Elle a démontré que beaucoup restait encore à faire.

Pourtant, dès 1948, l'Assemblée générale des Nations Unies avait institué un comité chargé de proposer l'établissement d'une Cour pénale internationale. Un projet de statut fut d'ailleurs adopté en 1953. Son examen fut néanmoins différé par l'Assemblée du fait de la guerre froide et du manque de consensus entre les États sur la définition de l'agression, définition sans laquelle il n'était pas envisageable de parvenir à un accord sur la création d'une cour permanente chargée de juger les crimes les plus graves. 
En décembre 1989, l'Assemblée générale demande à la Commission du droit international de reprendre ses travaux sur la création d'une Cour criminelle internationale. Un projet de statut fut remis à l'Assemblée générale en 1994. Après rapport d'un comité spécial sur le sujet, le Comité préparatoire pour la création d'une Cour criminelle internationale a mis au point un projet de texte en avril 1998. Une Conférence diplomatique de plénipotentiaires des Nations Unies a été convoquée à Rome du 15 juin au 17 juillet 1998. C'est par 120 voix pour, 7 contre et 21 abstentions que fut adopté le Statut portant création de la Cour pénale internationale(2).

\section{Une institution originale}

L'une des principales originalités de la Cour tient au fait à la fois qu'elle est la première juridiction pénale internationale permanente et le fruit d'un compromis entre les systèmes dits de Common Law et de droit romano-germanique (3). La Cour pénale internationale dont le siège a été fixé à La Haye (à l'instar de la Cour internationale de justice) est composée de quatre organes distincts : la présidence, les sections de jugement, le Bureau du procureur et le greffe. Elle est composée en tout et pour tout de 18 juges, élus pour neuf ans par l'Assemblée des États parties ${ }^{(4)}$.

\section{Tour d'horizon des organes de la Cour}

La Présidence (Presidency) de la Cour pénale internationale se compose de trois magistrats : le Président (the President), le Premier vice-président et le Second vice-président (the First and Second Vice-Presidents). Ces magistrats sont élus pour trois ans et rééligibles une fois. Conformément aux Statuts, la Présidence est chargée de la bonne administration de la Cour, à l'exception notable du Bureau du Procureur qui agit comme organe indépendant.

Une fois nommés, les juges sont répartis dans les trois sections principales : la Section préliminaire (the Pre-trial Division), la Section de première instance (the Trial Division), et la Section

(2) Le Statut est entré en vigueur le 2 juillet 2002, après que soixante États l'ont ratifié, conformément à l'Article 126 du Statut. Le Règlement de procédure et de preuve, les Éléments des crimes, le Règlement financier et règles de gestion financière et l'Accord sur les privilèges et immunités de la Cour ont été adoptés en septembre 2002. L'adoption du Statut du personnel et du Règlement de la Cour est intervenue respectivement en 2003 et 2004.

(3) Nombreux sont les juristes (francophones pour la plupart) qui ont fustigé la prééminence du système de Common Law sur le système romano-germanique au sein des tribunaux pénaux internationaux pour le Rwanda et la Yougoslavie.

(4) L'article 36 paragraphe 8 du Statut dispose : "Dans le choix des juges, les États parties tiennent compte de la nécessité d'assurer, dans la composition de la Cour : i) la représentation des principaux systèmes juridiques du monde [nous soulignons] ; (ii) une représentation géographique équitable ; et (iii) une représentation équitable des hommes et des femmes". 
des appels (the Appeals Division). Au sein de chaque section, les fonctions judiciaires de la Cour sont exercées par des chambres (chambers). La Section de première instance et la Section préliminaire sont chacune composées de six juges ayant l'expérience des procès pénaux. La Section des appels est, quant à elle, composée de 5 juges. II faut également préciser que les juges affectés à la Section des appels siègent exclusivement dans cette Section.

Le Bureau du Procureur (the Office of the Prosecutor) est un organe distinct de la Cour qui agit de manière indépendante. II est essentiellement chargé de recevoir les communications et tout renseignement concernant des crimes relevant de la compétence de la Cour, de les examiner, de conduire les enquêtes y afférentes et de soutenir l'accusation devant la Cour. Le Bureau est dirigé par le Procureur (the Prosecutor) assisté de procureurs adjoints (Deputy Prosecutors). Le Procureur est élu au scrutin secret par l'Assemblée des États parties, à la majorité absolue des membres de celle-ci. Les procureurs adjoints sont élus de la même façon sur une liste de candidats présentée par le Procureur. À moins qu'il ne soit décidé d'un mandat plus court au moment de leur élection, le Procureur et les procureurs adjoints exercent leurs fonctions pendant neuf ans et ne sont pas rééligibles.

Le Greffe de la Cour (the Registry) est dirigé par le Greffier (the Registrar) assisté d'un Greffier adjoint (Deputy Registrar). II est responsable des aspects non judiciaires de l'administration et du service de la Cour. Le Greffier est élu au scrutin secret par les juges à la majorité absolue. II est élu pour cinq ans et rééligible une fois. En outre, le Greffier a pour fonction de créer, au sein du Greffe, une Division d'aide aux victimes et aux témoins (Victims and Witnesses Unit). Cette division est chargée, en consultation avec le Bureau du Procureur, de conseiller et d'aider de toute manière appropriée, les témoins ou les victimes qui comparaissent devant la Cour ainsi que les autres personnes auxquelles les dépositions de ces témoins peuvent faire courir un risque, ainsi que de prévoir les mesures et les dispositions à prendre pour assurer leur protection et leur sécurité. Le personnel de la Division comprend des spécialistes de l'aide aux victimes de traumatismes, y compris de traumatismes consécutifs à des violences sexuelles.

\section{La confrontation de deux systèmes juridiques?}

Les juristes qui se sont penchés sur les travaux préparatoires et les Statuts de la Cour n'ont pas manqué de relever les nombreuses divergences qui sont apparues lors des discussions entre les tenants du système de Common Law et les partisans du système romano-germanique. Les règles de procédure et de preuve, notamment en ce qui concerne la phase préliminaire du jugement, ont été parmi les principales pierres d'achoppement. Si le principe accusatoire cher aux juristes de Common Law avait été retenu pour les tribunaux internationaux pour la Yougoslavie et le Rwanda, c'est un véritable " rééquilibrage " qui a été opéré dans le Statut de la CPI avec la création d'une chambre préliminaire chargée notamment de contrôler les preuves recueillies par les parties et de fournir une aide aux personnes mises en cause afin 
qu'elles puissent obtenir la coopération des États et assurer leur défense correctement(5). L'institution de la Chambre préliminaire introduit une dose d'inquisitoire ${ }^{(6)}$ bien connue des juristes civilistes. De la même manière, l'Article 75 du Statut prévoit un système d'indemnisation des victimes qui ressortit davantage au système romano-germanique qu'au système de Common Law et leur permet de percevoir un dédommagement pour le préjudice subi. Deux autres principes proches du système romano-germanique ont également été introduits, notamment une participation active de la victime au procès et le fait que les décisions de la Cour sont adoptées à la majorité sans qu'il ne soit possible pour les juges d'émettre des opinions individuelles séparées, comme cela est fréquent dans le système de Common Law et le système judiciaire international(7).

On le voit, les règles définies dans le cadre du Statut de la Cour pénale internationale tranchent avec celles adoptées pour les tribunaux pénaux internationaux qui portaient clairement la marque du système de Common Law, en particulier du système pénal américain. Pour autant, concilier les deux approches n'a pas été facile comme le montrent les divers documents rédigés à l'appui des réunions entre les représentants des États parties et la valse-hésitation qui a accompagné le choix des concepts de droit utilisés et de leur traduction dans les différentes langues de l'Organisation.

\section{Des vicissitudes de quelques concepts et de leur traduction}

Le premier élément qu'il convient de mentionner concernant les travaux préparatoires, les divers projets de Statut et le Statut lui-même est qu'ils ont été rédigés en anglais pour la plupart(8), puis traduits dans les langues officielles des Nations Unies, soit l'arabe, le chinois, l'espagnol, le français et le russe. On notera que dans le document onusien référencé sous la cote A/Conf.183/9, toutes les langues officielles de l'Organisation apparaissent comme langue originale. Cela s'explique par le fait que les différentes versions des instruments juridiques

(5) On trouvera une analyse comparative des compétences des tribunaux pénaux internationaux et de la CPI sur le site du Sénat [http://www.senat.fr/rap/r98-313/r98-31328.html].

(6) On remarquera, à cet égard, que la traduction du Rapport du Comité préparatoire pour la création d'une Cour criminelle internationale fait mention non pas d'une chambre préliminaire en français, mais bien d'une chambre d'instruction. C'est ce même terme qui avait été retenu dans la proposition française. Voir le document de travail présenté par la France, p.15.

(7) La règle est posée à l'Article 74 du Statut qui précise toutefois que : "S'il n'y a pas unanimité, la décision contient les vues de la majorité et de la minorité ".

(8) La principale exception concerne les documents présentés par les pays de langue française, la France notamment, qui étaient rédigés en français. 
importants que sont les conventions ou les traités ont la même valeur que le texte original conformément au principe d'égale authenticité qui prévaut au sein des organisations internationales ${ }^{(9)}$.

Un autre point d'intérêt concerne la dénomination en français de la Cour. Pendant tout le processus préparatoire, la Cour a été dénommée "Cour criminelle internationale ", comme l'attestent tous les documents référencés dans les archives de l'Organisation des Nations Unies. Oh! Surprise, elle est devenue "Cour pénale internationale " dans le texte final du Statut. Sans que l'on sache véritablement à quoi ce changement est dû, il semblerait que dans un premier temps les traducteurs aient opté pour la notion de "Cour criminelle internationale " par analogie avec la terminologie utilisée au Canada et du fait de sa proximité avec le terme anglais. Au cours des discussions successives et des projets qui en ont résulté, c'est l'optique française qui semble l'avoir emporté (l'adjectif pénal ayant, dans l'hexagone, progressivement remplacé celui de criminel considéré comme vieilli par certains). Cette hypothèse semble se confirmer à la lecture d'un article publié par l'avocat canadien André Sirois ${ }^{(10)}$ dans lequel il fustige le choix qui a été fait et défend la dénomination de "Cour criminelle " retenue dans un premier temps. Établissant une distinction entre les adjectifs " pénal " et " criminel ", le droit pénal incluant le droit criminel sans que l'inverse soit valable, André Sirois précise que dans la mesure où les tribunaux pénaux internationaux et, par analogie, la Cour pénale internationale ${ }^{(11)}$, sont appelés à juger des crimes et uniquement des crimes, il est plus correct et plus précis de parler de cour ou tribunal criminel que de cour ou tribunal pénal. À l'argument selon lequel l'adjectif " criminel " serait un anglicisme, André Sirois rétorque, à juste titre, que cette notion est parfaitement connue du droit français depuis bien longtemps ${ }^{(12)}$.

Si l'on ne peut que souscrire entièrement aux écrits de l'avocat québécois sur ce dernier point et sur la rigueur dont il convient de faire preuve à l'heure de traduire des concepts de droit, l'on doit également admettre l'idée que le droit et la traduction juridique tels qu'ils sont pratiqués dans les organisations internationales ne peuvent faire fi des considérations diplomatiques. Aussi ce n'est peut-être pas tant à une tendance à la transposition des traducteurs

(9) Cela ne signifie pas pour autant que les traductions sont approuvées par les États en même temps que le texte original, certaines traductions n'intervenant que bien plus tard. Dans le cas des Statuts de la Cour, on signalera néanmoins des dates différentes de mise en distribution des différentes versions et le fait que les traductions françaises et espagnol, notamment, ont fait l'objet de correctifs.

(10) Sirois, André : "La traduction et l'interprétation devant les tribunaux pénaux internationaux " in Actes du colloque international sur la traduction juridique : histoire, théorie(s) et pratique, Université de Genève, 17-19 février 2000, Genève, Suisse, p. 554-555.

(11) Lors d'une discussion informelle dans les locaux de l'Office des Nations Unies à Genève avec André Sirois à l'occasion de la mise à jour du glossaire de la Commission du droit international (CDI) et l'établissement du glossaire de la Cour pénale internationale, en collaboration avec Nicole Meeus, il est apparu que celui-ci appliquait le même raisonnement pour la dénomination de la CPI.

(12) Ibid. 


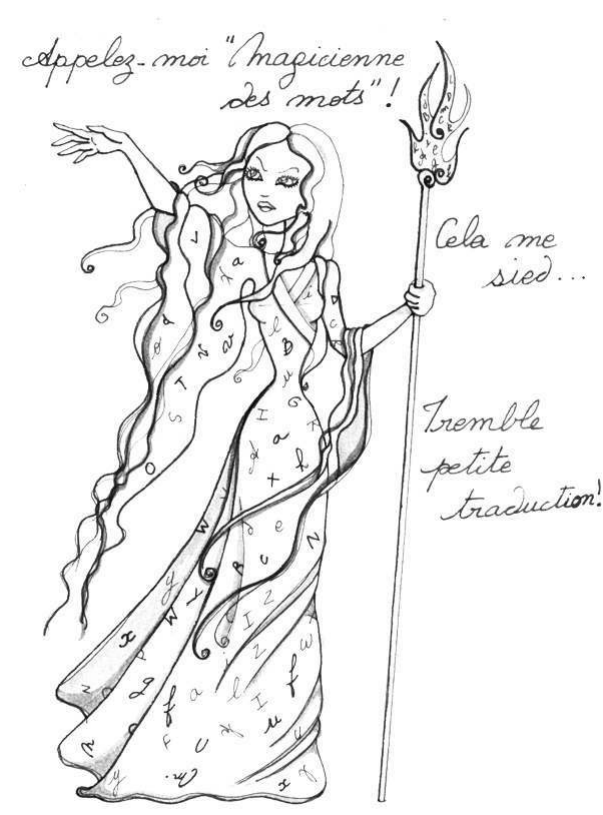

Illustration : Marlène Junius, http://alotoftralala.over-blog.com

français( ${ }^{(13)}$ mais davantage à des considérations diplomatiques que l'on doit cette dénomination car même si la notion de criminel n'a rien de péjoratif ou de dépréciatif en droit, il n'en est pas de même dans la langue courante et dans la langue diplomatique.

La notion de " situation ", qui apparaît à I'Article 14.1 du Statut, fournit un autre exemple intéressant de la manière dont les textes internationaux sont négociés et rédigés. En vertu de l'Article 14, "Tout État partie peut déférer au Procureur une "situation" dans laquelle un ou plusieurs des crimes relevant de la compétence de la Cour paraissent avoir été commis, et prier le Procureur d'enquêter sur cette "situation " en vue de déterminer si une ou plusieurs personnes particulières doivent être accusées de ces crimes ". Le lecteur averti remarquera d'emblée le caractère général de cette formulation qui n'a en soi rien de juridique. L'utilisation de termes généraux est fréquente dans les textes internationaux. En effet, il arrive souvent que les États parties ne parviennent pas à un accord sur certains points, ce qui oblige les rédacteurs à employer des termes très généraux qui peuvent engendrer une certaine ambiguïté et paraître pour le moins étrange si l'on se réfère aux systèmes juridiques nationaux.

La lecture des différents documents préparatoires montre que ce sont les notions de crimes et de cases qui avaient été retenues en anglais dans un premier temps et traduites par celles de "crimes " ou " affaires "(14). Au cours des discussions, plusieurs délégations ont critiqué ces choix et indiqué leur préférence pour le terme " situation " dans la mesure où, selon elles, une situation peut comporter plusieurs crimes contrairement à une " affaire ». L'emploi d'un terme général permet de limiter les possibilités d'interprétation et, par conséquent, de ne pas mettre en péril l'application du texte international. À partir du moment où le terme situation a été retenu en anglais, les traducteurs n'avaient d'autres choix que d'opter pour une traduction littérale sans chercher à expliciter le terme ${ }^{(15)}$. Les traducteurs travaillant dans les organisa-

(13) C'est ce qui ressort en filigrane de la critique d'André Sirois.

(14) La notion d'" affaires " (en traduction de cases) apparaît dans le Rapport du Comité préparatoire pour la création d'une cour criminelle internationale, Volume I.

(15) Une explicitation aurait pu consister à traduire le terme anglais situation par " un ou des crimes ". 
tions internationales le savent, ils doivent dans la mesure du possible conserver la structure du texte à traduire afin de garantir que les diverses versions linguistiques du texte pourront être lues en parallèle et comparées. De trop grandes divergences de formulations rendraient la comparaison malaisée.

Un autre cas remarquable concerne les notions d'" information " et de "poursuites "(16) que l'on retrouve dans les documents préparatoires et qui ont respectivement été remplacées dans la version finale du Statut par le terme "enquête " et le verbe " poursuivre "(17). Le même phénomène se reproduit, à l'inverse, pour la notion anglaise de mens rea ou élément moral que la loi exige pour que l'infraction soit constituée. Dans les documents préparatoires à la Conférence des plénipotentiaires ${ }^{(18)}$, les rédacteurs n'ont pas retenu la dénomination latine, lui préférant celle plus moderne de mental element. De manière pour le moins surprenante, les traducteurs ont choisi de réintroduire dans leur traduction le concept de mens rea, lequel n'est pas utilisé comme tel dans les pays francophones de tradition romano-germanique qui lui préfèrent la notion d'" élément psychologique ". C'est cette notion qui a été retenue dans la version finale du Statut. Le point commun entre toutes ces solutions est qu'elles sont suffisamment génériques pour ne pas être rattachées à un système juridique en particulier et qu'elles offrent l'avantage d'être relativement proches des concepts retenus dans la version anglaise. L'exemple le plus frappant à cet égard concerne la notion d'Elements of crimes, contenue dans l'Article 9, qui " aident la Cour à interpréter et appliquer les articles 6, 7 et 8 du Statut "(19). Dans tous les documents préparatoires, le syntagme anglais a été traduit, à juste titre, par "éléments constitutifs des crimes ", notion bien connue des juristes francophones. Or, dans la version finale du Statut, l'adjectif " constitutifs " a été supprimé pour " coller " davantage à la formulation anglaise. On pourra regretter le manque de précision de la traduction française, mais on se rappellera que dans le contexte international le caractère général de la formulation est une garantie que le texte sera accepté par tous et que l'interprétation qui en sera faite sera uniforme.

\section{Conclusion}

Le Statut de la Cour pénale internationale fourmille d'exemples de ces compromis qu'il a fallu trouver entre le système de Common Law et le système romano-germanique et des difficultés

(16) La version originale en anglais du Statut fait référence aux notions de investigation et de prosecution.

(17) Alors que la version anglaise utilise alternativement le substantif prosecution et le verbe to proceed, la version française ne contient que le verbe "poursuivre".

(18) Notamment l'additif au Rapport du comité préparatoire pour la création d'une cour criminelle internationale, p. 56.

(19) Éléments des crimes, ICC/ASP/1/3, p. 116. 
qui en ont résulté pour les traducteurs. Le système mis en place peut être qualifié de mixte voire de totalement nouveau dans la mesure où il mêle des procédures pénales différentes, des acteurs de cultures juridiques réputées peu conciliables. De fait, il est apparu difficile pour certaines délégations de dépasser leurs systèmes nationaux et de proposer une terminologie différente de celle à laquelle elles sont habituées(20).

La particularité du droit pénal international, qui est à la croisée des chemins entre le droit international et le droit pénal, n'est pas étrangère à ce phénomène. En effet, sauf à créer des concepts juridiques et, partant, une terminologie propre, les organisations internationales se voient souvent contraintes d'employer des concepts existants dans les droits nationaux. Tout le problème étant de savoir de quel droit ces concepts doivent être tirés. L'hégémonie américaine au niveau international a eu pour conséquence directe que la plupart des concepts juridiques retenus dans le contexte international proviennent du droit américain. Les choses ne sont malheureusement pas aussi tranchées s'agissant de la traduction en français des concepts juridiques anglais pour laquelle deux théories s'affrontent, les traducteurs québécois d'un côté, les traducteurs français de l'autre. Si l'on peut considérer comme normale la tendance de chacun à vouloir se référer à des notions ou des concepts connus, on ne peut que la regretter, dans une optique internationale, dans la mesure où elle ne prend pas réellement en compte le fait que le droit tel qu'il est conçu au niveau international est le fruit de compromis, parfois importants, qui favorisent l'émergence de règles nouvelles et partant, de concepts nouveaux qui s'affranchissent des règles nationales. C'est dans cette perspective, et non dans une optique " nationale " que les traductions réalisées au sein des organisations internationales doivent être envisagées et analysées.

veronique.sauron@unige.ch

Véronique-Anne Sauron a une double formation en droit et en traduction. Elle enseigne la traduction juridique à l'École de traduction et d'interprétation de l'Université de Genève et à l'Université de Grenoble III (Stendhal). Elle travaille en tant que traductrice pour diverses institutions internationales.

\section{Bibliographie}

ARAGONÉS LUMERAS M. ; SAURON, V. : "Stratégies normalisatrices et culture institutionnelle : un regard sur la traduction dans les organisations internationales ", communication présentée à l'occasion du 1er colloque de traduction spécialisée, La Havane, Cuba, 8-13 décembre 2008.

(20) Dans sa proposition, la France fait notamment référence au Parquet pour désigner l'accusation. 
Conférence diplomatique de plénipotentiaires des Nations Unies sur la création d'une Cour criminelle internationale, Rapport du comité préparatoire pour la création d'une cour criminelle internationale, additif (A/CONF. 183/2/Add.1), Nations Unies, New York, 14 avril 1998.

Finalized draft text of the Elements of Crimes, PCNICC/2000/1/Add.2, Preparatory Commission for the International Criminal Court.

Finalized draft text of the Rules of Procedure and Evidence, PCNICC/2000/1/Add.1, Preparatory Commission for the International Criminal Court.

Projet de statut de la Cour criminelle internationale, document de travail présenté par la France, Comité préparatoire pour la création d'une cour criminelle internationale, 12-30 août 1996 (A/AC. 249/L. 3), Nations Unies, New York.

Rapport du Comité préparatoire pour la création d'une cour criminelle internationale, Volumes I et II (Travaux du Comité préparatoire en mars-avril et août 1996), Assemblée générale, Documents officiels, cinquante et unième session, supplément $N^{\circ} 22(A / 51 / 22)$, Nations Unies, New York, 1996.

Rome Statute of the International Criminal Court, Final Act, A /Conf. 183/9, United Nations Diplomatic Conference of Plenipotentiaries on the Establishment of an International Criminal.

SIROIS, André : " La traduction et l'interprétation devant les tribunaux pénaux internationaux " in Actes du colloque international sur la traduction juridique : histoire, théorie(s) et pratique, Université de Genève, 17-19 février 2000, Genève, Suisse, p. 537-557. 